\title{
Cubital tunnel syndrome: A report of two cases
}

\author{
F E Suleman, MB ChB, FCRad(D)(SA), MMedRad(D) \\ Department of Radiology, University of Pretoria
}

M Velleman, MB ChB, FCRad(D)(SA), MMedRad(D)

Radiology Department, Little Company of Mary Medical Centre, Pretoria

Corresponding author: F Suleman (fesuleman@gmail.com)

\begin{abstract}
Cubital tunnel syndrome is the second most common peripheral neuropathy of the upper limb. This is due to the anatomy of the tunnel, the physiological changes that the nerve undergoes during elbow flexion, as well as pathological conditions that occur within the tunnel. We present two cases of ulnar neuropathy occurring at the level of the cubital tunnel, demonstrating that this entity may occur owing to an identifiable cause or may show only signal alteration without a visible cause on MRI.
\end{abstract}

S Afr J Rad 2012;16(2):77-78.

\section{Introduction}

Cubital tunnel syndrome occurs as a result of compression of the ulnar nerve between the medial epicondyle, the olecranon and the roof of the tunnel that is formed by the retinaculum which is also known as Osborne's band or the arcuate ligament. ${ }^{1,2}$ Physiological compression occurs during elbow flexion, but compression may also be the result of masses in the tunnel, including ganglions and bursae or synovitis or osteophytes. ${ }^{3}$ Rarely, compression has been attributed to the presence of an accessory muscle - the anconeus epitrochlearis. ${ }^{2,3}$ The ulnar nerve is also the most commonly injured nerve, owing to the relatively unprotected location of the nerve within the tunnel at the elbow. ${ }^{3}$

\section{Case report}

Two unrelated men aged 40 and 42 years, respectively, were referred for magnetic resonance imaging (MRI) on separate occasions, with elbow pain and suspected cubital tunnel syndrome. There was no history of work-related causality or injury in either case. MRI was requested to rule out any underlying mass lesion or anatomic variants that might have contributed to the development of an ulnar neuropathy at the level of the cubital tunnel.

\section{Patient 1}

A 40-year-old man revealed high signal on T2W (T2 weighted) MRI in a thickened ulnar nerve with no evidence of mass lesions or osteodegenerative changes. A normal cubital tunnel retinaculum was seen and no anatomic variants were noted. The rest of the joint was within normal limits (Fig. 1). A diagnosis of cubital tunnel syndrome of unknown aetiology was made.

\section{Patient 2}

A 42-year-old man demonstrated the presence of an olecranon spur as well as an anconeus epitrochlearis muscle on MRI scanning. Tendinosis of the triceps muscle was also present. T2WI also demonstrated high signal in the thickened ulnar nerve (Fig. 2). A diagnosis of cubital tunnel syndrome was made, with both the olecranon spur and the anconeus epitrochlearis muscle being implicated in the aetiology.

\section{Discussion}

Physiological compression of the ulnar nerve within the cubital tunnel occurs without neuropathy. Pathological compression gives rise to cubital tunnel syndrome. Possible causes include overuse, subluxation

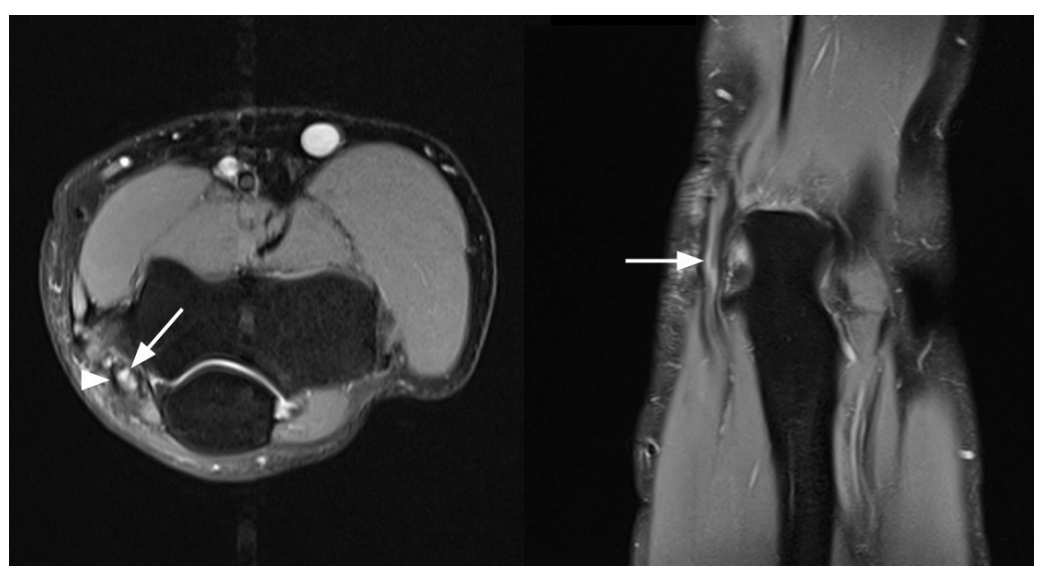

Fig. 1. Axial and coronal fat-suppressed MR images of the left elbow demonstrating increased signal in the thickened ulnar nerve (arrows). Note the normal retinaculum (arrowhead).

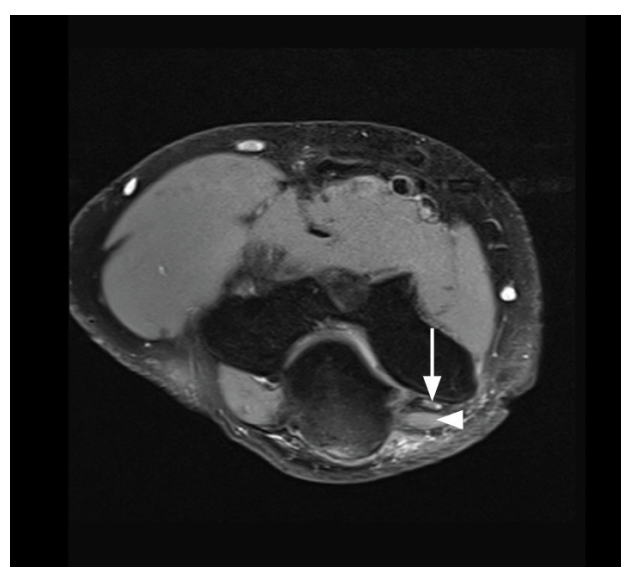

Fig. 2. Axial fat-suppressed MR image of the right elbow in patient 2, demonstrating the presence of the anconeus epitrochlearis muscle (arrowhead) and high signal in the thickened ulnar nerve (arrow). 


\section{CASE SERIES}

of the nerve, trauma including fractures around the elbow joint, osteophyte formation, soft-tissue masses and a thickened retinaculum. The presence of the anconeus epitrochlearis muscle is rarely implicated. The incidence is also reported to be higher in certain occupations especially those involving repetitive actions, prolonged flexion of the elbow and the use of vibrating tools. Diabetes and obesity are also predisposing factors. ${ }^{4}$

Patients may present with pain in the medial elbow but more commonly complain of sensory loss in the ulnar nerve distribution. Clinically, in long-standing cases, marked wasting of the small muscles of the hand on the ulnar side may be seen. ${ }^{4}$

The diagnosis is usually based on clinical findings and nerve conduction tests. Imaging is indicated only to exclude possible underlying causes of nerve entrapment. Radiographs of the elbow joint may show evidence of osteoarthritis or previous trauma, and ultrasound can be used to image the nerve itself but MRI is excellent for visualising changes in the signal of the nerve as well as changes in the nerve diameter while excluding any underlying masses or anatomical variants that may result in ulnar neuropathy. In a study of cadavers, O'Driscoll et al. ${ }^{5}$ classified the variants of the retinaculum into the following types: Type 0: absence of the retinaculum that predisposed to subluxation of the ulnar nerve

Type 1a: normal thin retinaculum that did not compress the nerve even in flexion

Type 1b: thickened retinaculum that was thought to predispose to chronic nerve compression

Type 3: replacement of the retinaculum by the anconeus epitrochealaris muscle.

MRI changes should be interpreted with caution, however, and the clinical picture should always be taken into consideration during the interpretation of images. In a study of the elbows of 60 asymptomatic patients, Husarik et al. ${ }^{3}$ found that $60 \%$ of subjects had increased signal in the ulnar nerve on fluid-sensitive MR images, and $23 \%$ of patients had the presence of an anconeus epitrochlearis muscle. The retinaculum was also found to be thickened in $8 \%$ of asymptomatic subjects. The increasing use of technology is also being implicated in the development of entrapment neuropathies of the upper limb. Ruess et al. ${ }^{1}$ reported on a study within their radiology department where a third of their radiologists sought medical attention during a 3-month period for symptoms in the upper limbs. All were found to be suffering from cubital tunnel syndrome, with one having carpal tunnel syndrome in addition. This implicated prolonged periods spent at the computer with widespread implementation of PACS, as well as administrative and teaching duties that involved computer use, as increasing the risk of development of entrapment syndromes. Prolonged elbow flexion involved in the use of hand-held dictation microphones and telephone receivers or mobile phones could also be contributing factors. Poor ergonomics of workstations were also thought to be a problem. They strongly recommended that radiology departments obtain professional advice from ergonomic experts and implement their recommendations for workstation designs.

\section{Conclusion}

MRI is well suited for demonstrating signal abnormality of the ulnar nerve in patients with cubital tunnel syndrome and also has the advantage of demonstrating causes such as osteophytes, soft-tissue masses, a thickened retinaculum and presence of an anconeus epitrochlearis muscle. Many factors relating to modern living, such as prolonged use of a computer mouse, telephone and, in certain occupations, dictation devices, could cause this syndrome, resulting in signal abnormality in the nerve without an obvious cause on MRI.

1. Ruess L, O'Connor SC, Cho KH, Hussein FH, Slaughter RC, Hedge A. Carpal tunnel syndrome and cubital tunnel syndrome: Work-related musculoskeletal disorders in four symptomatic radiologists. AJR 2003;181:37-41.

2. Andreisek G, Crook DW, Burg D, Marincek B, Weishaupt D. Peripheral neuropathies of the median, radial and ulnar nerves: MR imaging features. Radiographics 2006;26:1267-1287.

3. Husarik DB, Saupe N, Pfirmann CWA, Jost B, Hodler J, Zanetti M. Elbow nerves: MR findings in 60 asymptomatic subjects - normal anatomy, variants and pitfalls. Radiology 2009;252:148-156.

4. Cutts S. Cubital tunnel syndrome. Postgrad Med Journal 2007;83:28-31.

5. O Driscoll SW, Horii E, Carmichael SW, Morrey BF. The cubital tunnel and ulnar neuropathy. J Bone Joint Surg Br 1991;73:613-617 